\title{
Superconducting Films for Absorber-Coupled MKID Detectors for Sub-Millimeter and Far-Infrared Astronomy
}

\author{
Thomas R. Stevenson, Joseph S. Adams, Wen-Ting Hsieh, Samuel Harvey Moseley, Douglas E. Travers, \\ Kongpop U-yen, Edward J. Wollack, and Jonas Zmuidzinas
}

\begin{abstract}
We describe measurements of the properties, at dc, gigahertz, and terahertz frequencies, of thin $(10 \mathrm{~nm})$ aluminum films with $10 \Omega$ /square normal state sheet resistance. Such films can be applied to construct microwave kinetic inductance detector arrays for submillimeter and far-infrared astronomical applications in which incident power excites quasiparticles directly in a superconducting resonator that is configured to present a matched-impedance to the high frequency radiation being detected. For films $10 \mathrm{~nm}$ thick, we report normal state sheet resistance, resistance-temperature curves for the superconducting transition, quality factor and kinetic inductance fraction for microwave resonators made from patterned films, and terahertz measurements of sheet impedance measured with a Fourier Transform Spectrometer. We compare properties with similar resonators made from niobium $600 \mathrm{~nm}$ thick.
\end{abstract}

Index Terms-Aluminum, inductance, microwave measurements, niobium, $\mathbf{Q}$ factor, superconducting resonators.

\section{INTRODUCTION}

$\mathbf{M}$ ICROWAVE kinetic inductance detectors (MKIDs) [1] use the change in the kinetic inductance of a superconductor in response to pair-breaking radiation as a detection mechanism. Small changes in inductance can be detected as a change of resonance frequency and quality factor if the superconducting film is part of a microwave resonator. The detectors exhibit excess noise, apparently due to interaction with two-level systems in the dielectric of the microwave circuits [2], [3]; however, this noise problem has recently been mitigated by choice of readout technique [4]. Of the high-performance, low-temperature detector array technologies currently being developed, MKIDs may have the greatest promise for scaling to very large arrays ( $\sim 10^{5}$ detectors $)$ for application in a wide range of astronomical instruments. A variety of methods of radiation coupling have been investigated, including quasiparticle diffusion from an x-ray absorber [5], and phonon-mediated pair-breaking in dark matter detectors [6], [7]. For mil-

Manuscript received August 16, 2008. First published June 16, 2009; current version published July 10,2009 . This work was supported in part by an award under NASA's ROSES-APRA program.

T. R. Stevenson, J. S. Adams, W.-T. Hsieh, S. H. Moseley, D. E. Travers, K. U-yen, and E. J. Wollack are with NASA Goddard Space Flight Center, Greenbelt, MD 20771 USA (e-mail: Thomas.R.Stevenson@nasa.gov; Joseph.S. Adams@nasa.gov; wen-ting.hsieh-1@nasa.gov; harvey.moseley@nasa.gov; douglas.e.travers@nasa.gov; kongpop.u-yen-1@nasa.gov; edward.j.wollack@nasa.gov).

J. Zmuidzinas is with the Physics Department, California Institute of Technology, Pasadena, CA 91125 USA (e-mail: jonas@ caltech.edu).

Digital Object Identifier 10.1109/TASC.2009.2019661 limeter wave signals, radiation can be coupled into the device from an antenna, where it breaks Cooper pairs in the superconductor [8]. This technique works well at frequencies where the radiation can be coupled into the device using antennas and superconducting transmission lines. At frequencies above the superconducting gap of niobium, quality transmission lines are difficult to produce, so a different approach must be adopted.

For the 38-400 $\mu \mathrm{m}$ spectral region, we are investigating an approach in which optical signals directly illuminate a superconducting resonator chosen to have a surface-impedance and filling factor such that it presents an optimal matching impedance, about $157 \Omega$ /square, to the incoming wave at frequencies above its superconducting gap energy. The electromagnetic design of this absorber requires a normal state sheet resistance of $\sim 10 \Omega$ /square or greater. While thin films of superconducting alloys can show the desired values of sheet resistance, we focus in this paper on ultra-thin films of aluminum because more is known about the superconducting parameters for this material, including expected values of quasiparticle lifetime [9], [10]. From the resistivity-mean-free-path product for aluminum, with mean-free-path limited by film thickness, we estimated a required thickness of $7 \mathrm{~nm}$. This is significantly thinner than typical thicknesses for MKID x-ray or optical detectors (200-20 nm) [11]. For such thin films, the coherence length $\mid$, penetration depth $\angle$, and gap $\otimes$ of the material will be substantially modified from pure, bulk values $\iota_{0}=1600 \mathrm{~nm}$ and $L_{0}=16 \mathrm{~nm}$. We report on the deposition of $10 \mathrm{~nm}$ thick aluminum films with reproducible dc and terahertz sheet resistances, and compare measured and expected microwave properties of superconducting resonators made from this material. We also describe corresponding results for resonators of identical geometry made from $600 \mathrm{~nm}$ thick niobium films. Comparison of the resonance frequencies for the two sets of resonators allowed us to determine the kinetic inductance of the aluminum film independently from other parameters.

\section{DEVICE FABRICATION}

The aluminum films were deposited by electron-beam evaporation on bare silicon substrates that had been cleaned chemically, and with in-situ argon beam ion-milling. The base pressure was $35 \mu \mathrm{Pa}$. The deposition thickness was controlled with a crystal monitor, at a rate $\approx 0.015 \mathrm{~nm} / \mathrm{s}$. The final thickness, including native oxide, measured with a stylus profilometer was $9.5-11 \mathrm{~nm}$ for a shadow step. The $600 \mathrm{~nm}$ niobium films were deposited by planar magnetron sputtering onto silicon wafers coated with $100 \mathrm{~nm}$ of $\mathrm{SiO}_{2}$. 


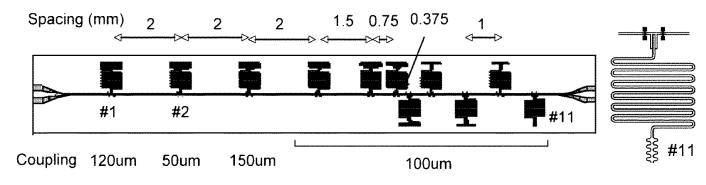

Fig. 1. Microwave resonator design. Overall chip size is $16.3 \mathrm{~mm} \times 2.3 \mathrm{~mm}$. Detail at right shows highest frequency resonator (\#11). Finger capacitors couple meandered CPW lines to the feedline. Each CPW is terminated in a short to ground via a "fish bone" section, with "spines" of various lengths to tune the resonance frequency (\#11 has all spines of minimum length).

Films were patterned into resonator arrays by reactive ion etching, in fluorine- or chlorine- based plasmas for $\mathrm{Nb}$ or $\mathrm{Al}$, respectively. The chip layout and resonator geometry is shown in Fig. 1. Eleven resonators were coupled to a common coplanar waveguide (CPW) feed-line, running the length of the chip, by interdigitated finger capacitors. The individual resonators each had a $7.43 \mathrm{~mm}$ long section of meandered CPW, followed by a $0.18 \mathrm{~mm}$ long section with four pairs of "spines" arranged in a "fish bone" pattern [12], and then a short to ground. The lengths of the spines varied between resonators to tune them to unique frequencies. The ground plane spacing and center conductor widths for the CPW lines were nominally 20 and $10 \mathrm{mi}$ crons respectively. Fig. 1 indicates the finger capacitor coupling lengths, and the physical spacing between resonators.

\section{SHEET RESISTANCE AT dc AND THz FREQUENCIES}

\section{A. Fourier Transform Spectrometer Measurements}

In the first of three aluminum depositions we made, we deposited unpatterned films of aluminum on $80 \mu \mathrm{m}$ thick high-resistivity silicon wafers as witness samples for Fourier Transform Spectrometer (FTS) measurements of transmission at frequencies spanning 0.6-7.5 THz. Measurements were made at temperatures of 295 and 5 K. Fig. 2 shows the $5 \mathrm{~K}$ data. From fits to the observed fringes, the sheet resistance was determined to be $R_{\mathrm{s}}=14.8 \Omega /$ square at $295 \mathrm{~K}$, and $10.5 \Omega /$ square at $5 \mathrm{~K}$. The residual resistance ratio was 1.41 . Assuming a resistivity-mean-free-path product for Al of $6.05 \times 10^{-16} \Omega^{2}$, we calculate an electronic mean-free-path for the film of $l=$ $5.8 \mathrm{~nm}$, somewhat less than its thickness.

\section{B. Four-Wire dc Resistance and Transition Temperature}

For a sample from a repeat deposition, we made four-wire resistance versus temperature measurements. The material had a residual resistance ratio $R R R=1.36$, a value similar to the first deposition, and exhibited a superconducting transition temperature $T_{\mathrm{c}}=1.78 \mathrm{~K}$ with a $10 \%-90 \%$ width of $5 \mathrm{mK}$.

\section{NiOBIUM MicrowaVE RESONATOR MEASUREMENTS}

We mounted the $\mathrm{Nb}$ resonator chip on a $\mathrm{Cu}$ block in an $\mathrm{Al}$ housing, with wire-bond connections to coaxial launchers and microstrip-to-CPW transition boards that we designed. Wirebonds across the CPW feedline were also used to tie different regions of ground plane together between resonators in order to suppress modes of undesired symmetry. We measured the microwave transmission $\mathrm{S}_{21}$ as a function of frequency with a vector-network-analyser with the sample in a liquid helium bath

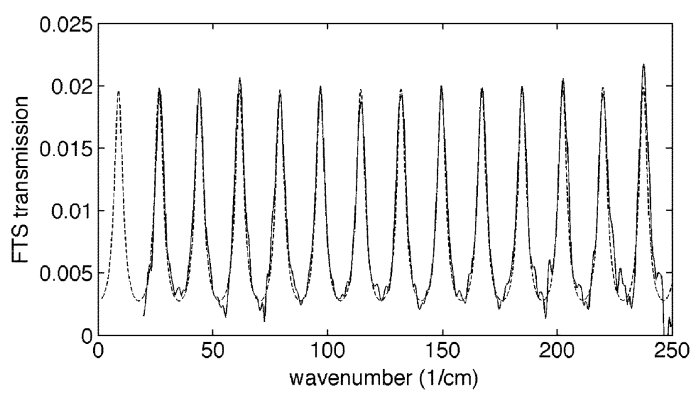

Fig. 2. FTS fringes at $5 \mathrm{~K}$, data (solid) and $R_{\mathrm{s}}=10.5 \Omega /$ square fit (dashed).

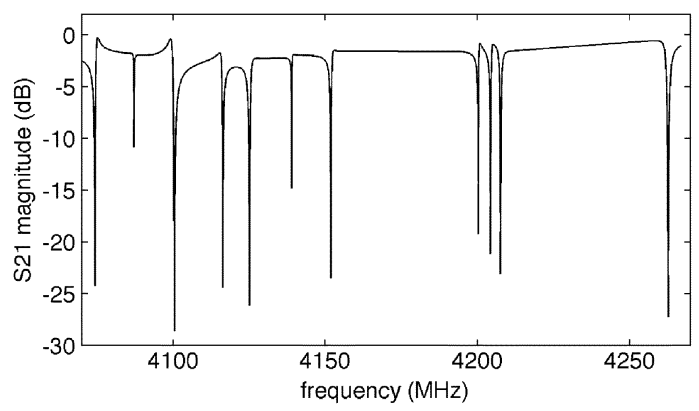

Fig. 3. Microwave transmission for the $\mathrm{Nb}$ resonator array at $\mathrm{T}=2.51 \mathrm{~K}$.

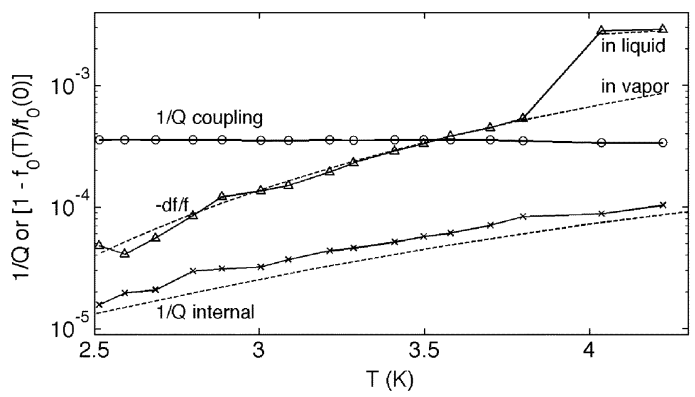

Fig. 4. Fractional shift in frequency, and inverse $Q$ values, versus temperature for $4.2628 \mathrm{GHz}$ niobium resonator (solid curves). Theoretical curves (dashed) use calculated kinetic inductance fraction (3\%) and $\mathrm{Nb}$ gap $\Delta=1.49 \mathrm{meV}$.

at temperatures $T=2.5-4.2 \mathrm{~K}$. A $-20 \mathrm{~dB}$ cold attenuator was placed on the microwave input to isolate the devices from room temperature radiation or noise. A cryogenic microwave amplifier [13] provided isolation and gain on the output. Fig. 3 shows the spectrum of eleven resonance frequencies, which spanned $4074-4263 \mathrm{MHz}$ at $2.51 \mathrm{~K}$. The resonance frequencies, which were designed to investigate a variety of tuning intervals, agreed with simulations to $\pm 7 \mathrm{MHz}$.

The temperature dependence of resonance frequency $f_{0}$ and quality factor $Q$ is shown for one mode (\#11) in Fig. 4. We extracted both the coupling and internal $Q$ by fitting the complex $S_{21}$ data. While pumping the helium bath, we observed a sudden drop in the resonance frequency by $0.2 \%$, the amount expected for the dielectric constant change between liquid and gaseous helium, as a vapor layer formed.

As shown in Fig. 4, the temperature dependence of the frequency agrees with the prediction of Mattis-Bardeen theory [14]. There are no free parameters in this fit: our $600 \mathrm{~nm}$ $\mathrm{Nb}$ films have $T_{\mathrm{c}} \approx 9.2 \mathrm{~K}$, hence $\otimes=1.49 \mathrm{meV}$, and we 


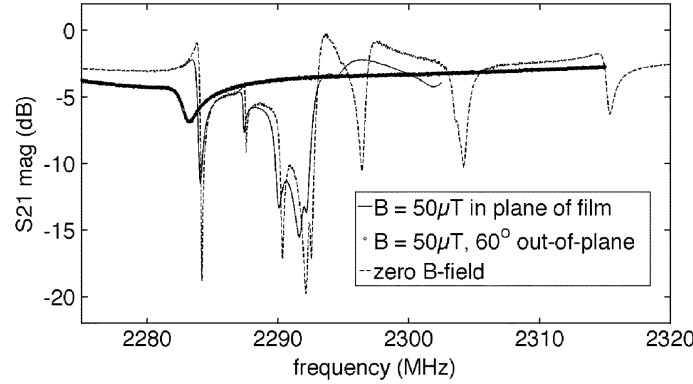

Fig. 5. $\mathrm{Al}$ array $S_{21}$ compared for different magnetic field orientations during cooling through $T_{\mathrm{c}}$, or with zero field, at $-81 \mathrm{dBm}$ feed power and $255 \mathrm{mK}$.

calculated the kinetic inductance fraction of our transmission line structures to be $\alpha=0.029$ using the method of [15], given that, from other microwave measurements, we know that our sputtered material has a penetration depth $\angle=80 \mathrm{~nm}$. The coupling $Q$, determined by the finger capacitance, was independent of $T$. The measured internal $Q$, reached values as high as 60,000 , and was within $30 \%$ of the theoretical prediction if we included a radiation-loss limit of $Q=170,000$.

\section{Aluminum MicrowaVe Resonator MEASUREMENTS}

We replaced the niobium resonator array in our microwave package with an aluminum version made in a third deposition run. We moved the package from the helium bath to the interior of the inner vacuum can of a ${ }^{3} \mathrm{He}$ refrigerator. We made multiple cooldowns with different components on the microwave input and output lines.

When we had direct dc connections to the chip, we could measure its dc $R-T$ curve. After correcting for the shunting effect of the silicon substrate at $T>200 \mathrm{~K}$, we found the resistance of the feed-line was $35.52 \mathrm{k} \Omega$ at $295 \mathrm{~K}$, and $25.91 \mathrm{k} \Omega$ at $4 \mathrm{~K}$. The $\mathrm{RRR}=1.37$ was again similar to our previous depositions. We measured $T_{\mathrm{c}}=1.755 \mathrm{~K}$ with a $10 \%-90 \%$ transition width of $16 \mathrm{mK}$. For a measurement current of $1 \mu \mathrm{A}$, there was a tail on the $R-T$ curve extending down to $1.4 \mathrm{~K}$, below which the device was fully superconducting. Between $1.40-1.75 \mathrm{~K}$ the resistance was unstable, fluctuating $\approx 200 \Omega$, which we attributed to the challenge of making good contact to the $10 \mathrm{~nm}$ film directly with aluminum wire-bonds.

To help ensure adequate cooling, we placed on the input line: a $20 \mathrm{~dB}$ attenuator in the $4 \mathrm{~K}$ bath, a section of stainless steel coaxial cable running in vacuum to the cold stage (outer conductor heat sunk at $1 \mathrm{~K}$ ), and a $3 \mathrm{~dB}$ attenuator on the cold stage. On the output, we inserted a bias-tee to provide (i) a dc connection to the cold stage, and (ii) an ac connection via stainless steel coaxial cable to the amplifier in the bath.

We initially made measurements in the Earth's magnetic field, but observed a strong dependence (Fig. 5) of $f_{0}$ and $Q$ on orientation during cooling through $T_{\mathrm{c}}$. If the field was not in the plane of the film, the resonances merged into one. We obtained the highest frequencies and Qs when we subsequently placed the dewar inside a magnetic shield.

Comparing thick $\mathrm{Nb}$ and thin $\mathrm{Al}$ resonators of the same geometry, the resonance frequencies for the $10 \mathrm{~nm} \mathrm{Al}$, shown in Fig. 6 , are lower by a factor of 1.84 , corresponding to a kinetic

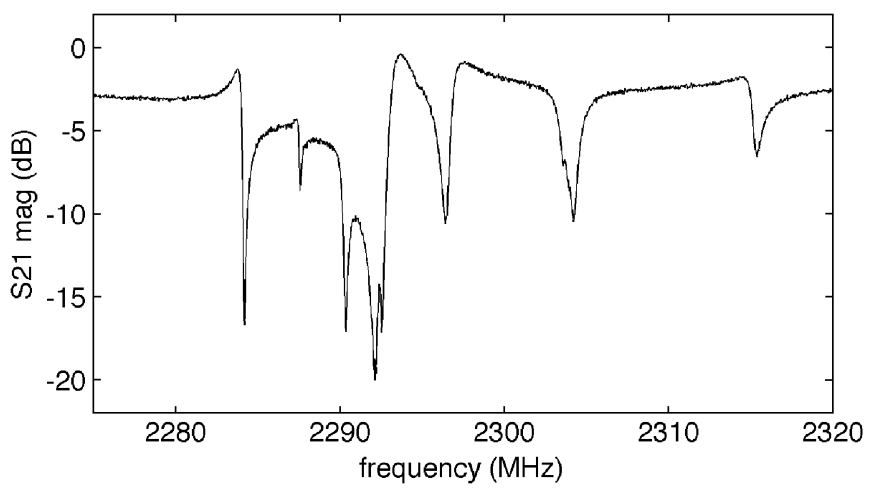

Fig. 6. Microwave transmission for aluminum array at $255 \mathrm{mK}$ in $B=0$.

inductance 2.49 times as large as the geometric inductance, instead of a ratio of just 0.03 for the $600 \mathrm{~nm} \mathrm{Nb}$. The kinetic inductance was $\alpha=71 \%$ of the total inductance. Up to ten of the 11 resonances were identifiable, but the fractional bandwidth spanned was reduced to $1.4 \%$ from $4.5 \%$ for the $\mathrm{Nb}$ array, causing overlap between the resonances. Electromagnetic modeling indicates that the reduced bandwidth arises from the use of the fish-bone structure for frequency tuning: for large kinetic inductance, the ground current will flow around the array of spines instead of following their edges.

The observed value of $\alpha$ matches theory. The reduced electronic mean-free-path $l$ should give modified coherence length and penetration depth:

$$
\begin{aligned}
\xi & =\sqrt{\xi_{0} l}=96 \mathrm{~nm}, \\
\lambda & =\lambda_{0} \sqrt{1+\xi_{0} / l}=270 \mathrm{~nm},
\end{aligned}
$$

where we have assumed $l=5.8 \mathrm{~nm}$ and $R_{\mathrm{s}}=10.5 \Omega$ /square from the FTS data as more accurate than values estimated from feed-line resistance, since line width was not measured precisely. One expects a kinetic surface inductance

$$
L_{K}=\mu_{0} \lambda^{2} / t=9.0 \mathrm{pH} / \text { square, }
$$

where $t=10 \mathrm{~nm}$ is the film thickness. From numerical integration of the Mattis-Bardeen integrals, and a measured value of $\Delta$ discussed below, one also expects that

$$
L_{K}=0.6442 R_{s} /(2 \Delta / \hbar)=8.4 \mathrm{pH} / \text { square. }
$$

The above two values are similar to the measured value

$$
L_{K}=L_{G} \alpha /(1-\alpha)=8.2 \mathrm{pH} / \mathrm{square},
$$

where $L_{\mathrm{G}}=3.3 \mathrm{pH}$ is the geometric inductance per square of center conductor calculated using the numerical method [15].

Fig. 7 shows that the temperature dependences of the resonance frequencies of all the modes are similar. Using the experimental value of $\alpha=0.71$, the observed frequency shifts match Mattis-Bardeen theory for $T>0.45 \mathrm{~K}$ if $\Delta=0.275 \mathrm{meV}$. The ratio of $2 \Delta /\left(k T_{\mathrm{c}}\right)=3.62$ is closer to the BCS value of 3.5 than the value 3.2 observed for thicker aluminum films [16]. For $T<0.45 \mathrm{~K}$, the frequency is more responsive to temperature than predicted, indicating that in our apparatus the electronic 


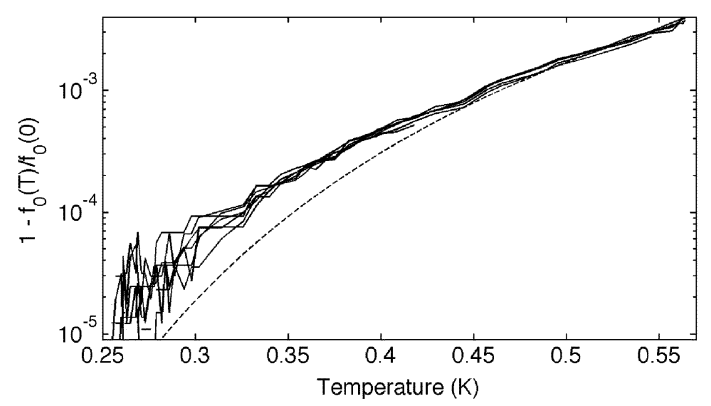

Fig. 7. Fractional shift in resonance frequency versus temperature for each aluminum resonator (solid curves). Theoretical curve (dashed) uses measured kinetic inductance fraction $\alpha=71 \%$ and fitted gap $\Delta=0.275 \mathrm{meV}$.

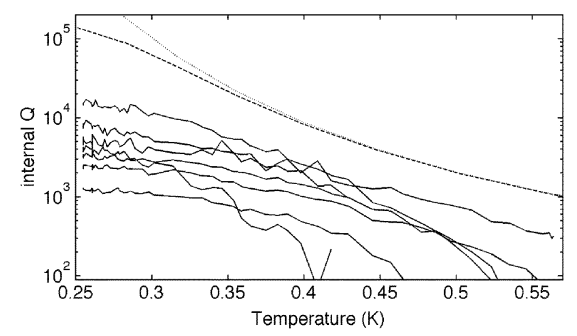

Fig. 8. Internal $\mathrm{Q}$ versus temperature for each aluminum resonator (solid curves). Theoretical curves without (dotted) and with (dashed) inclusion of radiation loss term equal to 170,000 value measured with niobium resonators.

temperature of the devices exceeded that of the $250 \mathrm{mK}$ cold stage by $\approx 30 \mathrm{mK}$.

We found it difficult to fit the internal Qs of the aluminum resonators since the reduced frequency spacing made the resonances overlap and interact. Fig. 8 shows the temperature dependence of internal Qs calculated from

$$
Q_{\text {int }}=\frac{f_{0}\left|d S_{21} / d f\right|}{2\left|S_{21}\right|} \text { at minima of }\left|S_{21}\right| \text {. }
$$

Equation (6) was evaluated for the seven clearest modes. As indicated in Fig. 8, the resulting Qs are 3-20 times lower than expected, but with a relative temperature dependence similar to theory. Simple models, incorporating Qs from (6) with the known resonator locations and couplings along the feed-line, tended to produce nulls in $S_{21}$ deeper and broader than observed. We speculate that the wire-bond air-bridges, insufficient in number, or in tenuous contact with the ultrathin films between the resonators, produced the difficult-to-model behavior. A preliminary attempt at more sophisticated 2-D simulations indicates the air-bridges are especially influential when $\alpha$ is high because the current flow is less localized.

We also studied the power dependence of the microwave transmission. The data in Figs. 6-8 were measured at a power level $\approx-91 \mathrm{dBm}$, approximately $1 \mathrm{pW}$, incident on the CPW feed line. The depth of the nulls in $S_{21}$ was insensitive to power at that level. As the power incident on the feedline was increased from 1 to $250 \mathrm{pW}$, the resonance frequency tended to decrease, but the depth of the null could increase or decrease, and eventually showed bifurcation behavior. Power dependence was several times larger when the devices were cooled in Earth's field parallel to film than for $B=0$.

\section{CONCLUSION}

We found it is practical to produce MKID devices from aluminum films as thin as $10 \mathrm{~nm}$. Our films had reproducible and stable normal state sheet resistances $\approx 10 \Omega$ /square, suitable for coupling of submillimeter radiation by direct absorption. The films were so thin as to have strongly modified superconducting parameters. Transition temperature and gap were increased compared to thicker films. Microwave behavior was sensitive to the magnetic field present during cooling through $T_{\mathrm{c}}$, and this required us to place the apparatus in a high-permeability magnetic shield. The $270 \mathrm{~nm}$ penetration depth $\lambda$ and $71 \%$ kinetic inductance fraction $\alpha$ were large. The magnitude and temperature dependence of the kinetic inductance agreed well with Mattis-Bardeen theory for a film in the local limit $(l \ll \xi<\lambda)$, rather than the extreme anomalous limit $(\lambda \ll \xi)$ that applies to thick aluminum.

\section{ACKNOWLEDGMENT}

The authors thank Ross Henry for FTS measurements of the aluminum films, and Christine Jhabvala and Mary Li for fabrication of the niobium resonators.

\section{REFERENCES}

[1] P. K. Day et al., "A broadband superconducting detector suitable for use in large arrays," Nature, vol. 425, pp. 817-821, 2003.

[2] J. Gao et al., "Experimental evidence for a surface distribution of twolevel systems in superconducting lithographed microwave resonators," Appl. Phys. Lett., vol. 92, pp. 152505-152507, 2008.

[3] J. Gao et al., "A semiempirical model for two-level system noise in superconducting microresonators," Appl. Phys. Lett., vol. 92, pp. 212504-212506, 2008.

[4] J. Gao et al., "Noise properties of superconducting coplanar waveguide microwave resonators," Appl. Phys. Lett., vol. 90, p. 102507, 2007.

[5] B. Mazin, B. Bumble, P. Day, M. Eckart, S. Golwala, J. Zmuidzinas, and F. Harrison, "Position sensitive X-ray spectrophotometer using microwave kinetic inductance detectors," Appl. Phys. Lett., vol. 89, p. 222507, 2006.

[6] M. Daal, B. Sadoulet, and J. Gao, "Kinetic inductance phonon sensors for the cryogenic dark matter search experiment," J. Low Temp. Phys., vol. 151, pp. 544-549, 2008.

[7] S. Golwala, J. Gao, D. Moore, B. Mazin, M. Eckart, B. Bumble, P. Day, H. G. LeDuc, and J. Zmuidzinas, "A WIMP dark matter detector using MKIDs,” J. Low. Temp. Phys., vol. 151, pp. 550-556, 2008.

[8] P. K. Day, H. G. LeDuc, A. Goldin, T. Vayonakis, B. A. Mazin, S. Kumar, J. Gao, and J. Zmuidzinas, "Antenna-coupled microwave kinetic inductance detectors," Nucl. Instrum. Methods Phys. Res. A, vol. 559, pp. 561-563, 2006.

[9] K. E. Gray, "Steady state measurements of the quasiparticle lifetime in superconducting aluminum," J. Phys. F: Metal Phys., vol. 1, pp. 290-308, 1971.

[10] C. Wilson, D. Prober, K. Segall, and L. Frunzio et al., Phys. Rev. B, vol. 69, p. 094524, 2004.

[11] B. A. Mazin, M. E. Eckart, B. Bumble, S. Golwala, P. K. Day, J. Gao, and J. Zmuidzinas, "Optical/UV and X-ray microwave kinetic inductance stip detectors," J. Low. Temp. Phys., vol. 151, pp. 537-543, 2008.

[12] K. U-yen, T. Stevenson, E. J. Wollack, C. Allen, W.-T. Hsieh, M. J. $\mathrm{Li}$, and J. Hicks, "Microwave resonator with multi-stage digital tuning technique for large-array frequency-multiplex readout systems," IEEE Trans. Microwave Theory and Techniques, 2008, submitted for publication.

[13] MITEQ model AFS 3-01000200-06-CR-6.

[14] D. C. Mattis and J. Bardeen, "Theory of the anomalous skin effect in normal and superconducting metals," Phys. Rev., vol. 11, no. 2, pp. 412-417, July 1958.

[15] W. H. Chang, "Numerical calculation of the inductances of a multi superconductor transmission-line system," IEEE Trans. Mag., vol. 17, p. 764, 1981 .

[16] A. Biondi and M. P. Garfunkel, "Millimeter wave absorption in superconducting aluminum. I. Temperature dependence of the energy gap," Phys. Rev., vol. 116, pp. 853-861, November 1959. 